\title{
Synthesis and styrene copolymerization of dimethyl, dimethoxy, and halogen ring-substituted isopropyl cyanophenylacrylates
}

Charles M. Rubert Pérez, Rachel L. Pride, Benjamin Y. Killam, Sara M. Rocus, William S. Schjerven and Gregory B. Kharas*

DePaul University, Chemistry and Biochemistry Department, 1110 West Belden Avenue, Chicago, IL 60614-3214

Contact:email:gkharas@depaul,edu

\begin{abstract}
Novel trisubstituted ethylenes, dimethyl, dimethoxy, and halogen ring-substituted isopropyl cyanophenylacrylates, $\mathrm{RPhCH}=\mathrm{C}(\mathrm{CN}) \mathrm{CO}_{2} \mathrm{CH}\left(\mathrm{CH}_{3}\right)_{2}$ (where $\mathrm{R}$ is 2,3-dimethyl, 2,4dimethyl, 2,5-dimethyl, 2,6-dimethyl, 3,4-dimethyl, 3,5-dimethyl, 2,3-dimethoxy, 2,4dimethoxy, 2,5-dimethoxy, 2-Br, 3-Br, 4-Br, 2-Cl, 3-Cl, 4-Cl, 2-F, 3-F, 4-F) were prepared and copolymerized with styrene. The monomers were synthesized by the piperidine catalyzed Knoevenagel condensation of ring-substituted benzaldehydes and isopropyl cyanoacetate and characterized by $\mathrm{CHN}$ elemental analysis, IR, ${ }^{1} \mathrm{H}$ - and ${ }^{13} \mathrm{C}-\mathrm{NMR}$. All the ethylenes were copolymerized with styrene in solution with radical initiation $(\mathrm{ABCN})$ at $70^{\circ} \mathrm{C}$. The composition of the copolymers was calculated from nitrogen analysis, and the structures were analyzed by IR, ${ }^{1} \mathrm{H}$ and ${ }^{13} \mathrm{C}-\mathrm{NMR}$, GPC, DSC, and TGA. Decomposition of
\end{abstract}


the copolymers in nitrogen occurred in two steps, first in the $219-500^{\circ} \mathrm{C}$ range with residue (0.9-5.6\% wt), which then decomposed in the $500-800^{\circ} \mathrm{C}$ range.

\section{Introduction}

Cyanophenylacrylates continue to attract attention as components and products of organic synthesis. Thus, methoxy ring-substituted methyl cyanophenylacrylates, MCPA was used in synthesis of pyridotriazines and triazolopyridines [1]. There are a number of applications of ethyl cyanophenylacrylates (ECPA), which include studies of catalysis [2] and as potential antimicrobial and antioxidant agents [3]. 2,4-Dimethoxyphenyl ECPA was used in design, synthesis and study of anticancer activity of novel benzothiazole analogues [4], in synthesis of thiazacridine derivatives as anticancer agents against breast and hematopoietic neoplastic cells [5] and in DABCO-catalyzed Knoevenagel condensation, using hydroxy ionic liquid as a promoter [6]. This ECPA was involved in catalysis study of N,N'-dialkylimidazolium dimethyl phosphates [7], in synthesis and study of antimicrobial activity of some cyanoacrylates [8], as well as in synthesis of antiproliferative active 2-aminobenzimidazole derivatives [9]. 2-Bromo ring-substituted ECPA is reported in heterocyclic synthesis of novel antimicrobial agents [10], norepinephrine transporter imaging agents [11], as well as, in synthesis and antiinflammatory activity of $\mathrm{N}$ and S-alkylated arylidene-thioxo-imidazolidinones [12]. 2Bromo and 4-bromo isopropyl cyanophenylacrylate (ICPA) was used in organocatalyzed enantioselective synthesis $[13,14]$, as well, in cyclodimerization of 1,1-dicyanoalkenes and arylidenecyanoacetates [15]. 3-Bromo ICPA is reported in reductive dimerization 
cyclization of arylmethylenecyanoacetates [16]. 2-Chloro ECPA was involved in application of prolinamide functionalized polyacrylonitrile fiber in catalysis [17] and in synthesis and studies of antifungal activities of novel polyheterocyclic spirooxindole derivatives [18]. 3-Chloro ECPA was reported in catalysis study [19] and DBU-promoted cascade annulation of nitroarylcyclopropane-1,1-dicarbonitriles and 3-aryl-2cyanoacrylates [20]. 4-Chloro ICPA was prepared via cyanuric chloride-mediated reactions involving condensation/cyano hydration/esterification [21]. It was also involved in cyclodimerization of arylidenecyanoacetate promoted by samarium diiodide [22]. 4Fluoro ICPA was reported in catalysis studies compartmentalization of incompatible polymers within metal-organic frameworks [23]. We have reported synthesis and styrene copolymerization of a number of dimethyl and dimethoxy ring-substituted esters of cyanophenylacrylates (CPA): methyl CPA [24, 25], ethyl CPA [26, 27], propyl CPA [28], and butyl CPA [29], as well as mono halogen phenyl-substituted CPA: methyl CPA [30], ethyl CPA [31], propyl CPA [32], butyl CPA [33], and isobutyl [34]. In continuation of our efforts in synthesis and copolymerization of ICPA compounds we have prepared dimethyl and dimethoxy ring-disubstituted and halogen ring monosubstituted ICPA compounds, $\mathrm{RPhCH}=\mathrm{C}(\mathrm{CN}) \mathrm{CO}_{2} \mathrm{CH}\left(\mathrm{CH}_{3}\right)_{2}$, where $\mathrm{R}$ is 2,3-dimethyl, 2,5-dimethyl, 2,6-dimethyl, 3,4-dimethyl, 2,3-dimethoxy, 2,4-dimethoxy, 2,5-dimethoxy, 2,6-dimethoxy 3,4-dimethoxy, 3,5-dimethoxy, 2-Br, 3-Br, 4-Br, 2-Cl, 3-Cl, 4-Cl, 2-F, 3-F, 4-F. To the best of our knowledge, except for syntheses of 2-Br, 4-Br, 4-Cl, and 4-F $(13,19,20,23)$ there have been no reports on either synthesis of these isopropyl cyanophenylacrylates, nor their copolymerization with styrene. 


\section{Experimental}

2,3-Dimethyl, 2,5-dimethyl, 2,6-dimethyl, 3,4-dimethyl, 2,3-dimethoxy, 2,4-dimethoxy, 2,5dimethoxy, 2,6-dimethoxy 3,4-dimethoxy, 3,5-dimethoxy, 2-Br, 3-Br, 4-Br, 2-Cl, 3-Cl, 4Cl, 2-F, 3-F, 4-F-benzaldehydes, isopropyl cyanoacetate, piperidine, styrene, 1,1'azobiscyclohexanecarbonitrile, (ABCN), and toluene supplied from Sigma-Aldrich Co., were used as received. Instrumentation is described in the first paper of this isopropyl esters' series [35].

\section{Synthesis of isopropyl cyanophenylacrylates}

The ICPA compounds were synthesized by Knoevenagel condensation [36] of a ringsubstituted benzaldehyde with isopropyl cyanoacetate, catalyzed by base, piperidine (Scheme 1).
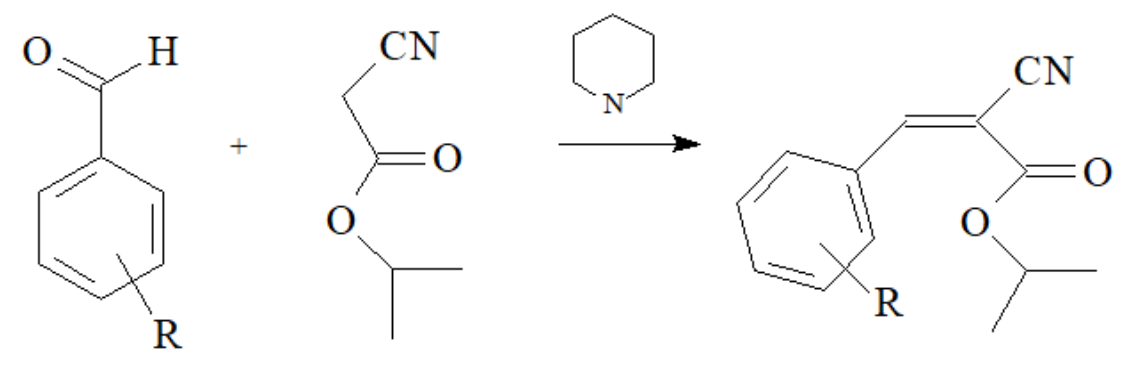

Scheme 1: Synthesis of isopropyl 2-cyano-3-phenyl-2-propenoates, where R is 2,3dimethyl, 2,4-dimethyl, 2,5-dimethyl, 2,6-dimethyl, 3,4-dimethyl, 3,5-dimethyl, 2,3dimethoxy, 2,4-dimethoxy 2,5-dimethoxy, 2-Br, 3-Br, 4-Br, 2-Cl, 3-Cl, 4-Cl, 2-F, 3-F, 4-F. 
The preparation procedure was essentially the same for all the monomers. In a typical synthesis, equimolar amounts of isopropyl cyanoacetate and an appropriate ring-substituted benzaldehyde were mixed in equimolar ratio in a $20 \mathrm{~mL}$ vial. A few drops of piperidine were added with stirring. The product of the reaction was isolated by filtration and purified by crystallization from 2-propanol. The condensation reaction proceeded smoothly, yielding products, which were purified by conventional techniques [35]. No stereochemical analysis of the novel ring-substituted ICPA was performed since no stereoisomers ( $E$ or/and $Z$ ) of known configuration were available.

\subsection{Isopropyl cyano(2,3-dimethylphenyl)acrylate}

Yield 92\%; mp $114^{\circ} \mathrm{C},{ }^{1} \mathrm{H}-\mathrm{NMR} \delta 8.3(\mathrm{~s}, 1 \mathrm{H}, \mathrm{CH}=), 7.8,7.2,7.0(\mathrm{~m}, 3 \mathrm{H}, \mathrm{Ph}), 5.1(\mathrm{~m}$, 1H, OCH), $2.3\left(\mathrm{~d}, 6 \mathrm{H}, \mathrm{CH}_{3}\right) 1.3\left(\mathrm{~d}, 6 \mathrm{H}, \mathrm{CH}\left(\mathrm{CH}_{3}\right)_{2}\right) ;{ }^{13} \mathrm{C}-\mathrm{NMR} \delta 166(\mathrm{C}=\mathrm{O}), 152(\mathrm{HC}=)$, 133, 131, 130, 126, $125(\mathrm{Ph}), 116(\mathrm{CN}), 104(\mathrm{C}=), 68(\mathrm{OCH}), 22\left(\mathrm{CH}\left(\mathrm{CH}_{3}\right)_{2}\right), 20,16$ $\left(\mathrm{CH}_{3}\right)$; FTIR $\left(\mathrm{cm}^{-1}\right)$ : 3028-2837 (m, C-H), $2226(\mathrm{~m}, \mathrm{CN}), 1728(\mathrm{~s}, \mathrm{C}=\mathrm{O}), 1596(\mathrm{C}=\mathrm{C})$, 1246 (s, C-O-C), 968, 842 (s, C-H out of plane). Anal. Calcd. for $\mathrm{C}_{15} \mathrm{H}_{17} \mathrm{NO}_{2}$ : C, 74.05; H, 7.04; N, 5.76; Found: C, 72.32; H, 6.96; N, 5.91.

\subsection{Isoropyl cyano(2,4-dimethylphenyl)acrylate}

Yield 84\%; mp 55 ${ }^{\circ} \mathrm{C},{ }^{1} \mathrm{H}-\mathrm{NMR} \delta 8.4(\mathrm{~s}, 1 \mathrm{H}, \mathrm{CH}=), 7.5,7.0,6.9$ (t, 3H, Ph), $5.1(\mathrm{~m}, 1 \mathrm{H}$, $\mathrm{OCH}), 2.3\left(\mathrm{~d}, 6 \mathrm{H}, \mathrm{CH}_{3}\right) 1.3\left(\mathrm{~d}, 6 \mathrm{H}, \mathrm{CH}\left(\mathrm{CH}_{3}\right)_{2}\right) ;{ }^{13} \mathrm{C}-\mathrm{NMR} \delta 166(\mathrm{C}=\mathrm{O}), 152(\mathrm{HC}=)$, 131, 130, 127, 126, $125(\mathrm{Ph}), 116(\mathrm{CN}), 104(\mathrm{C}=), 68(\mathrm{OCH}), 22\left(\mathrm{CH}\left(\mathrm{CH}_{3}\right)_{2}\right), 21,22$ $\left(\mathrm{CH}_{3}\right)$; FTIR $\left(\mathrm{cm}^{-1}\right):$ 3122-2802 (m, C-H), $2224(\mathrm{~m}, \mathrm{CN}), 1724(\mathrm{~s}, \mathrm{C}=\mathrm{O}), 1592(\mathrm{C}=\mathrm{C})$, 
1273, 1268 (s, C-O-C), 923, 839 (s, C-H out of plane). Anal. Calcd. for $\mathrm{C}_{15} \mathrm{H}_{17} \mathrm{NO}_{2}$ : C,

74.05; H, 7.04; N, 5.76; Found: C, 73.76; H, 6.96; N, 5.91.

\subsection{Isopropyl cyano(2,5-dimethylphenyl)acrylate}

Yield 87\%; mp $33^{\circ} \mathrm{C},{ }^{1} \mathrm{H}-\mathrm{NMR} \delta 8.5(\mathrm{~s}, 1 \mathrm{H}, \mathrm{CH}=), 7.5-7.0(\mathrm{~m}, 3 \mathrm{H}, \mathrm{Ph}), 5.3(\mathrm{~m}, 1 \mathrm{H}$, $\mathrm{OCH}), 2.3\left(\mathrm{~d}, 6 \mathrm{H}, \mathrm{CH}_{3}\right) 1.3\left(\mathrm{~d}, 6 \mathrm{H}, \mathrm{CH}\left(\mathrm{CH}_{3}\right)_{2}\right) ;{ }^{13} \mathrm{C}-\mathrm{NMR} \delta 166(\mathrm{C}=\mathrm{O}), 152(\mathrm{HC}=)$, 136, 133, 131, $130(\mathrm{Ph}), 116(\mathrm{CN}), 104(\mathrm{C}=), 68(\mathrm{OCH}), 22\left(\mathrm{CH}\left(\mathrm{CH}_{3}\right)_{2}\right), 21,20\left(\mathrm{CH}_{3}\right)$; FTIR $\left(\mathrm{cm}^{-1}\right)$ : 3052-2847 (m, C-H), $2224(\mathrm{~m}, \mathrm{CN}), 1724$ (s, C=O), 1558 (C=C), 1279 (s, C-O- $\mathrm{CH}_{3}$ ), 935, 844 (s, C-H out of plane). Anal. Calcd. for $\mathrm{C}_{15} \mathrm{H}_{17} \mathrm{NO}_{2}$ : C, 74.05; H, 7.04; N, 5.76; Found: C, 72.30; H, 7.02; N, 5.69.

\subsection{Isopropyl cyano(2,6-dimethylphenyl)acrylate}

Yield 91\%; mp $46^{\circ} \mathrm{C},{ }^{1} \mathrm{H}-\mathrm{NMR} \delta 8.5(\mathrm{~s}, 1 \mathrm{H}, \mathrm{CH}=), 7.5-7.0(\mathrm{~m}, 3 \mathrm{H}, \mathrm{Ph}), 5.3(\mathrm{~m}, 1 \mathrm{H}$, $\mathrm{OCH}), 2.3\left(\mathrm{~d}, 6 \mathrm{H}, \mathrm{CH}_{3}\right) 1.3\left(\mathrm{~d}, 6 \mathrm{H}, \mathrm{CH}\left(\mathrm{CH}_{3}\right)_{2}\right) ;{ }^{13} \mathrm{C}-\mathrm{NMR} \delta 166(\mathrm{C}=\mathrm{O}), 152(\mathrm{HC}=)$, 136, 133, 131, $130(\mathrm{Ph}), 116(\mathrm{CN}), 104(\mathrm{C}=), 68(\mathrm{OCH}), 22\left(\mathrm{CH}\left(\mathrm{CH}_{3}\right)_{2}\right), 21,20\left(\mathrm{CH}_{3}\right)$; FTIR $\left(\mathrm{cm}^{-1}\right)$ : 3052-2847 (m, C-H), $2224(\mathrm{~m}, \mathrm{CN}), 1724$ (s, C=O), 1558 (C=C), 1279 (s, C-O- $\mathrm{CH}_{3}$ ), 935, 844 (s, C-H out of plane). Anal. Calcd. for $\mathrm{C}_{15} \mathrm{H}_{17} \mathrm{NO}_{2}$ : C, 74.05; H, 7.04; N, 5.76; Found: C, 71.39; H, 6.92; N, 5.66.

\subsection{Isopropyl cyano(3,4-dimethylphenyl)acrylate}

Yield 79\%; mp $83^{\circ} \mathrm{C},{ }^{1} \mathrm{H}-\mathrm{NMR} \delta 8.6(\mathrm{~s}, 1 \mathrm{H}, \mathrm{CH}=), 8.2-7.0(\mathrm{~m}, 3 \mathrm{H}, \mathrm{Ph}), 5.3(\mathrm{~m}, 1 \mathrm{H}$, $\mathrm{OCH}), 2.3\left(\mathrm{~d}, 6 \mathrm{H}, \mathrm{CH}_{3}\right) 1.3\left(\mathrm{~d}, 6 \mathrm{H}, \mathrm{CH}\left(\mathrm{CH}_{3}\right)_{2}\right) ;{ }^{13} \mathrm{C}-\mathrm{NMR} \delta 166(\mathrm{C}=\mathrm{O}), 154(\mathrm{HC}=)$, 137, 136, 131, 130, $125(\mathrm{Ph}), 116(\mathrm{CN}), 104(\mathrm{C}=), 68(\mathrm{OCH}), 22\left(\mathrm{CH}\left(\mathrm{CH}_{3}\right)_{2}\right), 19\left(\mathrm{CH}_{3}\right)$; FTIR $\left(\mathrm{cm}^{-1}\right)$ : 3164-2850 (m, C-H), $2222(\mathrm{~m}, \mathrm{CN}), 1722(\mathrm{~s}, \mathrm{C}=\mathrm{O}), 1602(\mathrm{C}=\mathrm{C}), 1245(\mathrm{~s}$, 
C-O-CH ), 849 (s, C-H out of plane). Anal. Calcd. for $\mathrm{C}_{15} \mathrm{H}_{17} \mathrm{NO}_{2}: \mathrm{C}, 74.05 ; \mathrm{H}, 7.04 ; \mathrm{N}$,

5.76; Found: C, 72.83; H, 7.14; N, 5.69.

\subsection{Isopropyl cyano(3,5-dimethylphenyl)acrylate}

Yield $82 \%$; mp $72{ }^{\circ} \mathrm{C},{ }^{1} \mathrm{H}-\mathrm{NMR} \delta 8.2(\mathrm{~s}, 1 \mathrm{H}, \mathrm{CH}=), 7.3-7.0(\mathrm{~m}, 3 \mathrm{H}, \mathrm{Ph}), 5.3(\mathrm{~m}, 1 \mathrm{H}$, $\mathrm{OCH}), 2.4\left(\mathrm{~d}, 6 \mathrm{H}, \mathrm{CH}_{3}\right) 1.3\left(\mathrm{~d}, 6 \mathrm{H}, \mathrm{CH}\left(\mathrm{CH}_{3}\right)_{2}\right) ;{ }^{13} \mathrm{C}-\mathrm{NMR} \delta 166(\mathrm{C}=\mathrm{O}), 154(\mathrm{HC}=)$, 137, 136, 131, 130, $125(\mathrm{Ph}), 116(\mathrm{CN}), 104(\mathrm{C}=), 68(\mathrm{OCH}), 22\left(\mathrm{CH}\left(\mathrm{CH}_{3}\right)_{2}\right), 19\left(\mathrm{CH}_{3}\right)$; FTIR $\left(\mathrm{cm}^{-1}\right):$ 3164-2850 (m, C-H), $2224(\mathrm{~m}, \mathrm{CN}), 1720$ (s, C=O), $1610(\mathrm{C}=\mathrm{C}), 1249$ (s, C-O- $\mathrm{CH}_{3}$ ), 835 (s, C-H out of plane). Anal. Calcd. for $\mathrm{C}_{15} \mathrm{H}_{17} \mathrm{NO}_{2}$ : C, 74.05; H, 7.04; N, 5.76; Found: C, 72.66; H, 6.95; N, 5.67.

\subsection{Isopropyl cyano(2,3-dimethoxyphenyl)acrylate}

Yield 96\%; mp $66^{\circ} \mathrm{C},{ }^{1} \mathrm{H}-\mathrm{NMR} \delta 8.6(\mathrm{~s}, 1 \mathrm{H}, \mathrm{CH}=), 7.9-6.9(\mathrm{~m}, 3 \mathrm{H}, \mathrm{Ph}), 5.1(\mathrm{~m}, 1 \mathrm{H}$, $\mathrm{OCH}), 3.8\left(\mathrm{~d}, 6 \mathrm{H}, \mathrm{CH}_{3} \mathrm{O}\right), 1.3\left(\mathrm{~d}, 6 \mathrm{H}, \mathrm{CH}\left(\mathrm{CH}_{3}\right)_{2}\right) ;{ }^{13} \mathrm{C}-\mathrm{NMR} \delta 166(\mathrm{C}=\mathrm{O}), 152(\mathrm{HC}=)$, 152, 127, 126, $121(\mathrm{Ph}), 116(\mathrm{CN}), 111(\mathrm{C}=), 68(\mathrm{OCH}), 60,56\left(\mathrm{OCH}_{3}\right), 22\left(\mathrm{CH}\left(\mathrm{CH}_{3}\right)_{2}\right)$;

FTIR (cm $\left.{ }^{-1}\right):$ 3120-2790 (m, C-H), $2224(\mathrm{~m}, \mathrm{CN}), 1724(\mathrm{~s}, \mathrm{C}=\mathrm{O}), 1615(\mathrm{C}=\mathrm{C}), 1250$ (s, C-O-CH 3 ), 810, 762 (s, C-H out of plane). Anal. Calcd. for $\mathrm{C}_{15} \mathrm{H}_{17} \mathrm{NO}_{4}: \mathrm{C}, 65.44 ; \mathrm{H}$, 6.22; N, 5.09; Found: C, 63.89; H, 6.19; N, 4.95 .

\subsection{Isopropyl cyano(2,4-dimethoxyphenyl)acrylate}

Yield $87 \%$; mp $62^{\circ} \mathrm{C},{ }^{1} \mathrm{H}-\mathrm{NMR} \delta 8.6(\mathrm{~s}, 1 \mathrm{H}, \mathrm{CH}=), 7.6-6.9(\mathrm{~m}, 3 \mathrm{H}, \mathrm{Ph}), 5.1(\mathrm{~m}, 1 \mathrm{H}$, $\mathrm{OCH}), 3.8\left(\mathrm{~d}, 6 \mathrm{H}, \mathrm{CH}_{3} \mathrm{O}\right), 1.3\left(\mathrm{~d}, 6 \mathrm{H}, \mathrm{CH}\left(\mathrm{CH}_{3}\right)_{2}\right) ;{ }^{13} \mathrm{C}-\mathrm{NMR} \delta 166(\mathrm{C}=\mathrm{O}), 152(\mathrm{HC}=)$, 152, 131, 106, $98(\mathrm{Ph}), 116(\mathrm{CN}), 96(\mathrm{C}=), 68(\mathrm{OCH}), 56,55\left(\mathrm{OCH}_{3}\right), 22\left(\mathrm{CH}_{\left.\left(\mathrm{CH}_{3}\right)_{2}\right)}\right)$; FTIR (cm $\left.{ }^{-1}\right):$ 3982-2867 (m, C-H), $2224(\mathrm{~m}, \mathrm{CN}), 1723$ (s, C=O), $1607(\mathrm{C}=\mathrm{C}), 1286(\mathrm{~s}$, 
C-O-CH ), 842 (s, C-H out of plane). Anal. Calcd. for $\mathrm{C}_{15} \mathrm{H}_{17} \mathrm{NO}_{4}$ : C, 65.44; H, 6.22; N, 5.09; Found: C, 67.37; H, 6.64; N, 5.65.

\subsection{Isopropyl cyano(2,5-dimethoxyphenyl)acrylate}

Yield 77\%; mp $87^{\circ} \mathrm{C},{ }^{1} \mathrm{H}-\mathrm{NMR} \delta 8.7$ (s, $\left.1 \mathrm{H}, \mathrm{CH}=\right), 7.9-6.6(\mathrm{~m}, 3 \mathrm{H}, \mathrm{Ph}), 5.2(\mathrm{~m}, 1 \mathrm{H}$, $\mathrm{OCH}), 3.9\left(\mathrm{~d}, 6 \mathrm{H}, \mathrm{CH}_{3} \mathrm{O}\right), 1.3\left(\mathrm{~d}, 6 \mathrm{H}, \mathrm{CH}\left(\mathrm{CH}_{3}\right)_{2}\right) ;{ }^{13} \mathrm{C}-\mathrm{NMR} \delta 166(\mathrm{C}=\mathrm{O}), 152(\mathrm{HC}=)$, 152, 131, 106, $\left.98(\mathrm{Ph}), 116(\mathrm{CN}), 96(\mathrm{C}=), 68(\mathrm{OCH}), 56,55\left(\mathrm{OCH}_{3}\right), 22\left(\mathrm{CH}_{\left(\mathrm{CH}_{3}\right.}\right)_{2}\right)$; FTIR $\left(\mathrm{cm}^{-1}\right)$ : 3095-2807 (m, C-H), $2222(\mathrm{~m}, \mathrm{CN}), 1718(\mathrm{~s}, \mathrm{C}=\mathrm{O}), 1611(\mathrm{C}=\mathrm{C}), 1264(\mathrm{~s}$, C-O-CH 3 ), 854 (s, C-H out of plane). Anal. Calcd. for $\mathrm{C}_{15} \mathrm{H}_{17} \mathrm{NO}_{4}$ : C, 65.44; H, 6.22; N, 5.09; Found: C, 65.51; H, 6.31; N, 5.06.

\subsection{Isopropyl cyano(2-bromophenyl)acrylate}

Yield 91\%; mp 55.8 ${ }^{\circ} \mathrm{C},{ }^{1} \mathrm{H}$ NMR $\delta 8.7(\mathrm{~s}, 1 \mathrm{H}, \mathrm{CH}=), 8.3-7.2(\mathrm{~m}, 4 \mathrm{H}, \mathrm{Ph}), 5.2(\mathrm{~m}, 1 \mathrm{H}$, $\mathrm{CH}), 1.4\left(\mathrm{~d}, 6 \mathrm{H}, \mathrm{CH}_{3}\right) ;{ }^{13} \mathrm{C} \mathrm{NMR} \delta 166(\mathrm{C}=\mathrm{O}), 152(\mathrm{HC}=), 137,133,131,130,128(\mathrm{Ph})$, $116(\mathrm{CN}), 106(\mathrm{C}=), 68(\mathrm{CH}), 22\left(\mathrm{CH}_{3}\right) ;$ FTIR $\left(\mathrm{cm}^{-1}\right): 3015-2836(\mathrm{~m}, \mathrm{C}-\mathrm{H}), 2226(\mathrm{~m}$, CN), 1726 (s, C=O), 1594 (C=C), 1253 (s, C-O-CH3), 766-741 (s, C-H out of plane). Anal. Calcd. for $\mathrm{C}_{13} \mathrm{H}_{12} \mathrm{BrNO}_{2}$ : C, 53.08; H, 4.11; N, 4.76; Found: C, 52.79; H, 4.42; N, 5.03 .

\subsection{Isopropyl cyano(3-bromophenyl)acrylate}

Yield 68\%; mp 85.3 ${ }^{\circ} \mathrm{C},{ }^{1} \mathrm{H}$ NMR $\delta 8.2(\mathrm{~s}, 1 \mathrm{H}, \mathrm{CH}=), 8.1-7.3(\mathrm{~m}, 4 \mathrm{H}, \mathrm{Ph}), 5.2(\mathrm{~m}, 1 \mathrm{H}$, $\mathrm{CH}), 1.8\left(\mathrm{~d}, 6 \mathrm{H}, \mathrm{CH}_{3}\right) ;{ }^{13} \mathrm{C} \mathrm{NMR} \delta 162(\mathrm{C}=\mathrm{O}), 153(\mathrm{HC}=), 137,134,131,123(\mathrm{Ph}), 115$ $(\mathrm{CN}), 104(\mathrm{C}=), 68(\mathrm{CH}), 22\left(\mathrm{CH}_{3}\right)$; FTIR $\left(\mathrm{cm}^{-1}\right)$ : 3038-2869 (m, C-H), $2223(\mathrm{~m}, \mathrm{CN})$, 
$1724(\mathrm{~s}, \mathrm{C}=\mathrm{O}), 1607(\mathrm{C}=\mathrm{C}), 1248\left(\mathrm{~s}, \mathrm{C}-\mathrm{O}-\mathrm{CH}_{3}\right), 788,750$ (s, C-H out of plane). Anal.

Calcd. for $\mathrm{C}_{13} \mathrm{H}_{12} \mathrm{BrNO}_{2}$ : C, 53.08; H, 4.11; N, 4.76; Found: C, 51.37; H, 4.21; N, 4.81.

\subsection{Isopropyl cyano(4-bromophenyl)acrylate}

Yield $88 \%$; mp $108.8^{\circ} \mathrm{C},{ }^{1} \mathrm{H}$ NMR $\delta 8.3(\mathrm{~s}, 1 \mathrm{H}, \mathrm{CH}=), 8.0-6.2(\mathrm{~m}, 4 \mathrm{H}, \mathrm{Ph}), 5.3(\mathrm{~m}, 1 \mathrm{H}$, $\mathrm{CH}), 1.5\left(\mathrm{~d}, 6 \mathrm{H}, \mathrm{CH}_{3}\right) ;{ }^{13} \mathrm{C} \mathrm{NMR} \delta 165(\mathrm{C}=\mathrm{O}), 154(\mathrm{HC}=), 133,132,131,128(\mathrm{Ph}), 116$

$(\mathrm{CN}), 114(\mathrm{C}=), 68(\mathrm{CH}), 22\left(\mathrm{CH}_{3}\right)$; FTIR $\left(\mathrm{cm}^{-1}\right):$ 3016-2807 (m, C-H), $2226(\mathrm{~m}, \mathrm{CN})$,

1717 (s, C=O), 1241 (s, C-O-C), 818 (s, C-H out of plane). Anal. Calcd. for

$\mathrm{C}_{13} \mathrm{H}_{12} \mathrm{BrNO}_{2}$ : C, 53.08; H, 4.11; N, 4.76; Found: C, 52.39; H, 4.34; N, 4.82.

\subsection{Isopropyl cyano(2-chlorophenyl)acrylate}

Yield 78\%; ${ }^{1} \mathrm{H}$ NMR $\delta 8.7(\mathrm{~s}, 1 \mathrm{H}, \mathrm{CH}=), 8.3-7.3(\mathrm{~m}, 4 \mathrm{H}, \mathrm{Ph}), 5.3(\mathrm{~m}, 1 \mathrm{H}, \mathrm{CH}), 1.2(\mathrm{~d}$, $\left.6 \mathrm{H}, \mathrm{CH}_{3}\right) ;{ }^{13} \mathrm{C} \mathrm{NMR} \delta 164(\mathrm{C}=\mathrm{O}), 152(\mathrm{HC}=), 137,132,131,130,127(\mathrm{Ph}), 116(\mathrm{CN})$, $116(\mathrm{C}=), 68(\mathrm{CH}), 22\left(\mathrm{CH}_{3}\right)$; FTIR $\left(\mathrm{cm}^{-1}\right): 3072-2815(\mathrm{~m}, \mathrm{C}-\mathrm{H}), 2226(\mathrm{~m}, \mathrm{CN}), 1730(\mathrm{~s}$, $\mathrm{C}=\mathrm{O}), 1609$ (C=C), 1248 (s, C-O-C), 760 (s, C-H out of plane). Anal. Calcd. for $\mathrm{C}_{13} \mathrm{H}_{12} \mathrm{ClNO}_{2}$ : C, 62.53; H, 4.84; N, 5.61; Found: C, 62.49; H, 4.93; N, 5.81.

\subsection{Isopropyl cyano(3-chlorophenyl)acrylate}

Yield 84\%; mp 159C ${ }^{\circ}$, ${ }^{1} \mathrm{H}$ NMR $\delta 8.2(\mathrm{~s}, 1 \mathrm{H}, \mathrm{CH}=), 8.0-7.2(\mathrm{~m}, 4 \mathrm{H}, \mathrm{Ph}), 5.1(\mathrm{~m}, 1 \mathrm{H}$, $\mathrm{CH}), 1.3\left(\mathrm{~d}, 6 \mathrm{H}, \mathrm{CH}_{3}\right) ;{ }^{13} \mathrm{C} \mathrm{NMR} \delta 162(\mathrm{C}=\mathrm{O}), 154(\mathrm{HC}=), 135,133,128(\mathrm{Ph}), 116$ $(\mathrm{CN}), 104(\mathrm{C}=), 68(\mathrm{CH}), 22\left(\mathrm{CH}_{3}\right)$; FTIR $\left(\mathrm{cm}^{-1}\right)$ : 3026-2817 (m, C-H), $2223(\mathrm{~m}, \mathrm{CN})$, 1699 (s, C=O), 1597 (C=C), 1268 (s, C-O-C), 748 (s, C-H out of plane). Anal. Calcd. for $\mathrm{C}_{13} \mathrm{H}_{12} \mathrm{ClNO}_{2}$ : C, 62.53; H, 4.84; N, 5.61; Found: C, 62.69; H, 4.96; N, 5.68. 


\subsection{Isopropyl cyano(4-chlorophenyl)acrylate}

Yield 75\%; mp 113.7C $\mathrm{C}^{\circ}{ }^{1} \mathrm{H}$ NMR $\delta 8.2(\mathrm{~s}, 1 \mathrm{H}, \mathrm{CH}=), 8.0-7.2(\mathrm{~m}, 4 \mathrm{H}, \mathrm{Ph}), 5.3(\mathrm{~m}, 1 \mathrm{H}$, $\mathrm{CH}), 1.2\left(\mathrm{~d}, 6 \mathrm{H}, \mathrm{CH}_{3}\right) ;{ }^{13} \mathrm{C} \mathrm{NMR} \delta 166(\mathrm{C}=\mathrm{O}), 154(\mathrm{HC}=), 138,132,129(\mathrm{Ph}), 116$

$(\mathrm{CN}), 114(\mathrm{C}=), 68(\mathrm{CH}), 22\left(\mathrm{CH}_{3}\right)$; FTIR $\left(\mathrm{cm}^{-1}\right)$ : 3016-2891 (m, C-H), $2225(\mathrm{~m}, \mathrm{CN})$, 1731 (s, C=O), 1585 (C=C), 1222 (s, C-O-C), 815, 766 (s, C-H out of plane). Anal.

Calcd. for $\mathrm{C}_{13} \mathrm{H}_{12} \mathrm{ClNO}_{2}$ : C, 62.53; H, 4.84; N, 5.61; Found: C, 61.47; H, 4.97; N, 5.49.

\subsection{Isopropyl cyano(2-fluorophenyl)acrylate}

Yield 97\%; mp 59.5 ${ }^{\circ} \mathrm{C} ;{ }^{1} \mathrm{H}$ NMR $\delta 8.5$ (s, $\left.1 \mathrm{H}, \mathrm{CH}=\right), 8.4-7.0(\mathrm{~m}, 4 \mathrm{H}, \mathrm{Ph}), 5.3(\mathrm{~m}, 1 \mathrm{H}$, $\mathrm{CH}), 1.3\left(\mathrm{~d}, 6 \mathrm{H}, \mathrm{CH}_{3}\right) ;{ }^{13} \mathrm{C} \mathrm{NMR} \delta 164(\mathrm{C}=\mathrm{O}), 142,131,130,125,119(\mathrm{Ph}), 116(\mathrm{CN})$, $104(\mathrm{C}=), 68(\mathrm{CH}), 22\left(\mathrm{CH}_{3}\right)$; FTIR $\left(\mathrm{cm}^{-1}\right): 3096-2834(\mathrm{~m}, \mathrm{C}-\mathrm{H}), 2222(\mathrm{~m}, \mathrm{CN}), 1720(\mathrm{~s}$, $\mathrm{C}=\mathrm{O}), 1578(\mathrm{C}=\mathrm{C}), 1228$ (s, C-O-C), 820 (s, C-H out of plane). Anal. Calcd. for

$\mathrm{C}_{13} \mathrm{H}_{12} \mathrm{FNO}_{2}$ : C, 66.94; H, 5.19; N, 6.01; Found: C, 65.80; H, 5.36; N, 6.47.

\subsection{Isopropyl cyano(3-fluorophenyl)acrylate}

Yield $82 \%$; mp $87.7^{\circ} \mathrm{C} ;{ }^{1} \mathrm{H}$ NMR $\delta 8.3(\mathrm{~s}, 1 \mathrm{H}, \mathrm{CH}=), 7.9-7.2(\mathrm{~m}, 4 \mathrm{H}, \mathrm{Ph}), 5.3(\mathrm{~m}, 1 \mathrm{H}$, $\mathrm{CH}), 1.3\left(\mathrm{~d}, 6 \mathrm{H}, \mathrm{CH}_{3}\right) ;{ }^{13} \mathrm{C} \mathrm{NMR} \delta 166(\mathrm{C}=\mathrm{O}), 154(\mathrm{HC}=), 162,135,131(\mathrm{Ph}), 116$ $(\mathrm{CN}), 104(\mathrm{C}=), 68(\mathrm{CH}), 22\left(\mathrm{CH}_{3}\right)$; FTIR $\left(\mathrm{cm}^{-1}\right)$ : 3174-2754 (m, C-H), $2222(\mathrm{~m}, \mathrm{CN})$, 1717 (s, C=O), 1612 (C=C), 1268 (s, C-O-C), 835, 763 (s, C-H out of plane). Anal. Calcd. for $\mathrm{C}_{13} \mathrm{H}_{12} \mathrm{FNO}_{2}$ : C, 66.94; H, 5.19; N, 6.01; Found: C, 64.64; H, 5.20; N, 6.03.

\subsection{Isopropyl cyano(4-fluorophenyl)acrylate}

Yield 92\%; mp 91.2 ${ }^{\circ} \mathrm{C} ;{ }^{1} \mathrm{H}$ NMR $\delta 8.2(\mathrm{~s}, 1 \mathrm{H}, \mathrm{CH}=), 8.1-7.1(\mathrm{~m}, 4 \mathrm{H}, \mathrm{Ph}), 5.3(\mathrm{~m}, 1 \mathrm{H}$, $\mathrm{CH}), 1.3\left(2,6 \mathrm{H}, \mathrm{CH}_{3}\right) ;{ }^{13} \mathrm{C} \mathrm{NMR} \delta 166(\mathrm{C}=\mathrm{O}), 154(\mathrm{HC}=), 134,131,129,117(\mathrm{Ph}), 116$ 
$(\mathrm{CN}), 108(\mathrm{C}=), 68(\mathrm{CH}), 22\left(\mathrm{CH}_{3}\right) ;$ FTIR $\left(\mathrm{cm}^{-1}\right)$ : 3142-2782 (m, C-H), $2242(\mathrm{~m}, \mathrm{CN})$, 1728 (s, C=O), 1608 (C=C), 1214 (s, C-O-C), 810 (m, C-H out of plane). Anal. Calcd. for $\mathrm{C}_{13} \mathrm{H}_{12} \mathrm{FNO}_{2}$ : C, 66.94; H, 5.19; N, 6.01; Found: C, 65.78; H, 5.09; N, 5.87.

\section{Copolymerization}

Copolymers of the ST and the ICPA monomers were prepared in 25-mL glass screw cap vials at $\mathrm{ST} / \mathrm{ICPA}=3(\mathrm{~mol})$ the monomer feed using $0.12 \mathrm{~mol} / \mathrm{L}$ of $\mathrm{ABCN}$ at an overall monomer concentration $2.44 \mathrm{~mol} / \mathrm{L}$ in $10 \mathrm{~mL}$ of toluene. The copolymerization was conducted at $70^{\circ} \mathrm{C}$. After a predetermined time, the mixture was cooled to room temperature, and precipitated dropwise in methanol. The conversion of the copolymers was kept between below $20 \%$ to minimize compositional drift. The composition of the copolymers was determined based on the nitrogen content. The ST-ICPA copolymers were characterized by nitrogen elemental analysis, FTIR, ${ }^{1} \mathrm{H}$ - and ${ }^{13} \mathrm{C}-\mathrm{NMR}$ spectroscopies. Thermal behavior was studied by DSC and TGA.

Copolymerization (Scheme 1) of ST and the ring-substituted ICPA resulted in formation of copolymers (Table 1) with weight-average molecular masses $13.5-69.5 \mathrm{kD}$. Since ICPA monomers do not homopolymerize, the most likely structure of the copolymers would be isolated ICPA monomer units $(n=1)$ alternating with short ST $(\mathrm{m}=1-4)$ sequences (Scheme 2). The copolymers prepared in the present work are all soluble in ethyl acetate, THF, DMF and $\mathrm{CHCl}_{3}$ and insoluble in methanol, ethyl ether, and petroleum ether. According to the nitrogen elemental analysis, between 13.5 and 31.2 
mol\% of ICPA monomer is present in the copolymers, which is indicative of relatively high reactivity of the monomers towards ST.

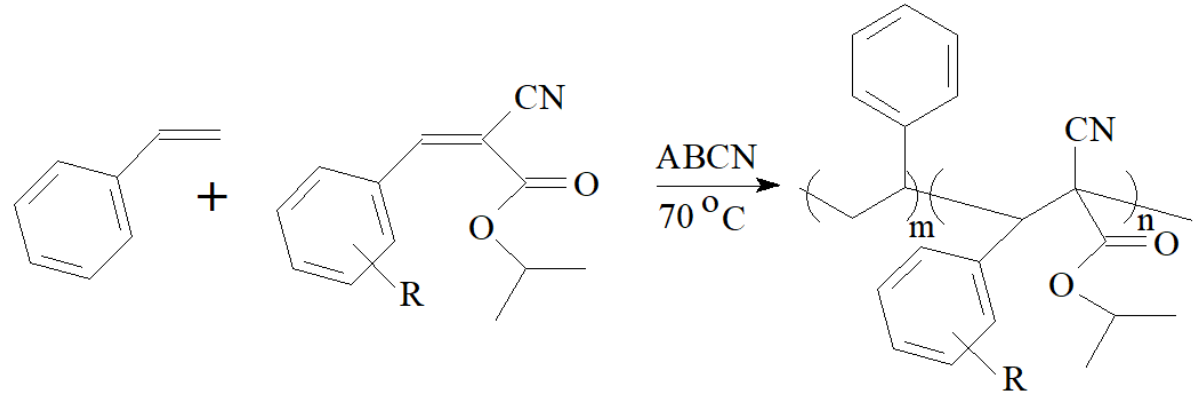

Scheme 2. ST-ICPA copolymer synthesis, R = 2,3-dimethyl, 2,4-dimethyl, 2,5-dimethyl, 2,6-dimethyl, 3,4-dimethyl, 3,5-dimethyl, 2,3-dimethoxy, 2,4-dimethoxy 2,5-dimethoxy, 2$\mathrm{Br}, 3-\mathrm{Br}, 4-\mathrm{Br}, 2-\mathrm{Cl}, 3-\mathrm{Cl}, 4-\mathrm{Cl}, 2-\mathrm{F}, 3-\mathrm{F}, 4-\mathrm{F}$.

\begin{tabular}{|c|c|c|c|c|c|c|c|c|c|}
\hline & & & & & & $T G A$ & & & \\
\hline $\mathrm{R}$ & $\begin{array}{l}\text { Yield }^{\mathrm{a}} \\
\mathrm{wt} \%\end{array}$ & $\begin{array}{l}\mathrm{N} \\
\mathrm{wt} \%\end{array}$ & $\begin{array}{l}\text { ICP } \\
\text { A in } \\
\text { pol., } \\
\text { mol } \\
\%\end{array}$ & $\begin{array}{l}\mathrm{M}_{\mathrm{w}} \\
\mathrm{kD}\end{array}$ & $\begin{array}{l}\mathrm{T}_{\mathrm{g}} \\
{ }^{\circ} \mathrm{C}\end{array}$ & $\begin{array}{l}\text { Onset } \\
\text { of } \\
\text { dec., } \\
{ }^{\circ} \mathrm{C}\end{array}$ & $\begin{array}{l}10 \% \\
\mathrm{wt} \\
\text { loss, } \\
{ }^{\circ} \mathrm{C}\end{array}$ & $\begin{array}{l}50 \% \\
\mathrm{wt} \\
\text { loss, } \\
{ }^{\circ} \mathrm{C}\end{array}$ & $\begin{array}{l}\text { Residu } \\
\text { e at } \\
500^{\circ} \mathrm{C}, \\
\mathrm{wt} \%\end{array}$ \\
\hline $2,3-\left(\mathrm{CH}_{3}\right)_{2}$ & 12.1 & 2.44 & 23.9 & 63.2 & 127 & 279 & 306 & 338 & 4.9 \\
\hline $2,4-\left(\mathrm{CH}_{3}\right)_{2}$ & 13.3 & 1.97 & 18.2 & 56.3 & 128 & 287 & 318 & 343 & 3.8 \\
\hline $2,5-\left(\mathrm{CH}_{3}\right)_{2}$ & 11.4 & 2.96 & 31.1 & 52.5 & 131 & 286 & 312 & 346 & 3.9 \\
\hline $2,6-\left(\mathrm{CH}_{3}\right)_{2}$ & 13.3 & 1.54 & 13.5 & 62.8 & 136 & 291 & 321 & 369 & 4.6 \\
\hline
\end{tabular}




\begin{tabular}{|l|l|l|l|l|l|l|l|l|l|}
\hline $3,4-\left(\mathrm{CH}_{3}\right)_{2}$ & 17.2 & 2.73 & 27.8 & 59.8 & 129 & 295 & 299 & 374 & 5.1 \\
\hline $3,5-\left(\mathrm{CH}_{3}\right)_{2}$ & 11.7 & 2.54 & 25.2 & 62.2 & 133 & 282 & 331 & 359 & 4.4 \\
\hline $2,3-\left(\mathrm{CH}_{3} \mathrm{O}\right)_{2}$ & 13.7 & 2.53 & 27.2 & 57.5 & 131 & 297 & 308 & 352 & 4.5 \\
\hline $2,4-\left(\mathrm{CH}_{3} \mathrm{O}\right)_{2}$ & 12.4 & 2.26 & 23.2 & 62.3 & 125 & 297 & 309 & 353 & 5.6 \\
\hline $2,5-\left(\mathrm{CH}_{3} \mathrm{O}\right)_{2}$ & 16.4 & 2.27 & 23.3 & 61.4 & 132 & 290 & 309 & 357 & 3.9 \\
\hline $2-\mathrm{Br}$ & 12.3 & 2.94 & 29.5 & 46.2 & 162 & 257 & 312 & 341 & 2.9 \\
\hline $3-\mathrm{Br}$ & 16.9 & 2.58 & 27.4 & 68.9 & 166 & 269 & 320 & 348 & 4.7 \\
\hline $4-\mathrm{Br}$ & 18.2 & 2.59 & 28.1 & 65.5 & 161 & 271 & 312 & 340 & 2.9 \\
\hline $2-\mathrm{Cl}$ & 17.2 & 2.93 & 31.2 & 42.5 & 161 & 267 & 314 & 342 & 3.2 \\
\hline $3-\mathrm{Cl}$ & 18.3 & 2.86 & 30.6 & 20.0 & 111 & 230 & 206 & 369 & 0.9 \\
\hline $4-\mathrm{Cl}$ & 19.2 & 2.73 & 30.1 & 50.4 & 127 & 219 & 308 & 351 & 3.5 \\
\hline $2-\mathrm{F}$ & 10.3 & 3.03 & 30.0 & 62.2 & 158 & 258 & 313 & 337 & 1.1 \\
\hline $3-\mathrm{F}$ & 11.8 & 2.88 & 28.6 & 69.5 & 155 & 260 & 306 & 333 & 1.4 \\
\hline $4-\mathrm{F}$ & 12.4 & 2.65 & 27.0 & 46.3 & 156 & 244 & 304 & 335 & 1.5 \\
\hline
\end{tabular}

${ }^{\mathrm{a} C o n d i t i o n s: ~ S T / I C A A: ~} 3$ (mol) / Toluene $/ 70^{\circ} \mathrm{C} / 5$ hrs.

\section{Structure and Thermal Properties}

The structure of ST- ICPA copolymers was characterized by IR and NMR spectroscopy. A comparison of the spectra of the monomers, copolymers and polystyrene shows, that the reaction between the trisubstituted ethylenes and styrene is a copolymerization. IR spectra of the copolymers show overlapping bands in $3250-2700 \mathrm{~cm}^{-1}$ region 
corresponding to $\mathrm{C}-\mathrm{H}$ stretch vibrations. The bands for the ICPA monomer unit are 22312225 (w, CN), 1740-1722 (s, C=O), and 1252-1220 $\mathrm{cm}^{-1}$ (m, C-O). Benzene rings of both monomers show ring stretching bands at 1511-1462 and 1521-1462 $\mathrm{cm}^{-1}$ as well as a doublet 781-671 $\mathrm{cm}^{-1}$, associated with $\mathrm{C}-\mathrm{H}$ out of plane deformations. These bands can be readily identified in styrene copolymers with trisubstituted ethylene monomers containing cyano and carbonyl electron withdrawing groups.

The ${ }^{1} \mathrm{H}-\mathrm{NMR}$ spectra of the ST- ICPA copolymers show a broad double peak in a 6.0-8.0 ppm region corresponding to phenyl ring protons. A resonance at 5.4-4.9 ppm is assigned to the methine protons of isopropyl group. A broad resonance at 4.2-3.8 ppm is methoxy protons, 3.8-2.0 ppm is assigned to the methine protons of ICPA, and methine and methylene protons of ST monomer unit close to the propenoate unit, which are more subjected to deshielding than the ones in polystyrene. The low and high field components of the signal are associated with ICPA monomer unit in head-to-tail and head-to-head structures. A broad resonance peak in 0.9-2.4 ppm range is attributed to the methine and methylene protons of styrene monomer sequences, as well as to methyl groups of isopropyl ester and alkyl-Ph protons of ICPA. The ${ }^{13} \mathrm{C}-\mathrm{NMR}$ spectra also support the suggested skeletal structure of the copolymers. Thus, the assignment of the peaks is as follows: 167-160 ppm (C=O), 157-130 ppm (quaternary carbons of both phenyls), 118112 ppm (CN), 60-50 ppm (methine, quaternary carbons and alkoxy ICPA carbons), 4745 ppm (ST methine), and 44-40 ppm (ST methylene), 36-14 ppm alkyl carbons of ICPA. The copolymers prepared in the present work are all soluble in ethyl acetate, THF, DMF and $\mathrm{CHCl} 3$ and insoluble in methanol, ethyl ether, and petroleum ether. 
All the copolymers were amorphous and show no crystalline DSC endotherm on repeated heating and cooling cycles. Table 2 shows glass transition values for the ST-ICPA copolymers prepared in this work with no correlation to the size and position of the ICPA ring substitution apparently due to non-uniform composition, monomer unit distribution, and/or molecular weight and MWD. A single Tg was observed for all the copolymers with values $111-166^{\circ} \mathrm{C}$. Information on thermal stability of the copolymers (Table 2) was obtained from thermogravimetric analysis (Table 2). Decomposition of the copolymers in nitrogen occurred in two steps, first in the $219-500^{\circ} \mathrm{C}$ range with residue $(0.9-5.6 \% \mathrm{wt})$, which then decomposed in the $500-800^{\circ} \mathrm{C}$ range. The decomposition products were not analyzed in this study, and the mechanism has yet to be investigated.

\section{Conclusion}

Novel dimethyl, dimethoxy, and halogen ring-substituted isopropyl cyanophenylacrylates were prepared and copolymerized with styrene. The compositions of the copolymers were calculated from nitrogen analysis and the structures were analyzed by IR, $\mathrm{H}^{1}$ and ${ }^{13} \mathrm{C}-\mathrm{NMR}$. The thermal gravimetric analysis indicated that the copolymers decompose in in two steps, first in the $219-500^{\circ} \mathrm{C}$ range with residue $(0.9-5.6 \% \mathrm{wt})$, which then decomposed in the $500-800^{\circ} \mathrm{C}$ range.

\section{Acknowledgments}

The authors are grateful to acknowledge that the project was partly supported by the Chicago Society of Coating Technology (CSCT). 


\section{References}

1. Zaki MEA, Fathalla OA, Swelam SA, Aly HF (2004) Synthesis of pyrido[2,1c][1,2,4]triazine, 1,2,4-triazolo[4,3-a]pyridine and 2-(pyrazolyl)nicotinonitrile and their effect on Biomphalaria alexandrina snail enzymes. Acta Poloniae Pharmaceutica 61: $55-64$.

2. Burate PA, Javle BR, Desale PH, Kinage AK (2019) Amino acid amide based ionic liquid as an efficient organo-catalyst for solvent-free Knoevenagel condensation at room temperature. Catalysis Letters 149(9): 2368-2375.

3. Medyouni R, Hamdi N, Ben Said R, Al-Ayed AS, Zagrouba F (2013) Clean procedure and DFT study for the synthesis of 2-amino-3-ethoxycarbonyl-4-(aryl)4H-pyrano-[3,2-c]-chromene-5-ones derivatives: A novel class of potential antimicrobial and antioxidant agents. Journal of Chemistry 2013: 1-9.

4. Hassan AY, Sarg MT, Hussein EM (2019) Design, synthesis and anticancer activity of novel benzothiazole analogues. Journal of Heterocyclic Chemistry 56(4): 14371457.

5. Moacyr JB, De Melo R, Wanessa LB, De Sena, RO, De Moura, Iris TT et al. (2017) Synthesis and anticancer evaluation of thiazacridine derivatives reveals new selective molecules to hematopoietic neoplastic cells. Combinatorial Chemistry \& High Throughput Screening 20(8): 713.

6. Meng D, Qiao Y, Wang X, Wen W, Zhao S (2018) DABCO-catalyzed Knoevenagel condensation of aldehydes with ethyl cyanoacetate using hydroxy ionic liquid as a promoter. RSC Advances 8(53): 30180-30185. 
7. Brica S, Freimane L, Kulikovska L, Zicmanis A (2017) N,N'-dialkylimidazolium dimethyl phosphates - promising media and catalysts at the same time for condensation reactions. Chemical Science International Journal 19(4): 1-9.

8. Bhuiyan M, Mosharef H, Rahman KM, Alam MA, Mahmud M (2013) Microwave assisted Knoevenagel condensation: synthesis and antimicrobial activities of some a-cyanoacrylates. Pakistan Journal of Scientific and Industrial Research, Series A: Physical Sciences 56(3): 131-137.

9. Synthesis and antiproliferative activity in vitro of new 2-aminobenzimidazole derivatives. Reaction of 2-arylideneaminobenzimidazole with selected nitriles containing active methylene group. Nowicka, Anna; Liszkiewicz, Hanna; Nawrocka, Wanda P.; Wietrzyk, Joanna; Kempinska, Katarzyna; Drys, Andrzej. Central European Journal of Chemistry (2014), 12(10), 1047-1055.

10. N-(furan-2-ylmethyl)-cyanoacetamide in heterocyclic synthesis: synthesis of novel antimicrobial agents encompassing furan, pyridine, chromene, and chromenopyridine moieties. Farag, Awatef A. Pharma Chemica (2015), 7(11), 130-141.

11. Synthesis and biological evaluation of trans-3-phenyl-1-indanamines as potential norepinephrine transporter imaging agents. McConathy, Jonathan; Owens, Michael J.; Kilts, Clinton D.; Malveaux, Eugene J.; Votaw, John R.; Nemeroff, Charles B.; Goodman, Mark M. Nuclear Medicine and Biology (2005), 32(6), 593-605.

12. Synthesis and anti-inflammatory activity of some new N and S-alkylated arylidenethioxo-imidazolidinones. Santos, L. C.; Mourao, R. H. V.; Uchoa, F. T.; Silva, T. G.; 
Malta, D. J. N.; Moura, R. O.; Lima, M. C. A.; Galdino, S. L.; Pitta, I. R.; Barbe, J. Heterocyclic Communications (2005), 11(5), 433-440.

13. Organocatalyzed Enantioselective Synthesis of 2-Amino-4H-chromene Derivatives. Ramireddy, Naresh; Abbaraju, Santhi; Ding, Derong; Arman, Hadi; Zhao, John C.-G. Journal of Heterocyclic Chemistry (2017), 54(1), 677-691.

14. Organocatalyzed enantioselective synthesis of 2-amino-5-oxo-5,6,7,8-tetrahydro-4Hchromene-3-carboxylates. Ramireddy, Naresh; Abbaraju, Santhi; Zhao, Cong-Gui. Tetrahedron Letters (2011), 52(50), 6792-6795.

15. Cyclodimerization of 1,1-dicyanoalkenes and arylidenecyanoacetates promoted by ytterbium diiodide. Su, W. K.; Yang, B. B. Journal of the Indian Chemical Society (2003), 80(3), 163-165.

16. Metallic ytterbium-mediated reductive dimerization cyclization of arylmethylenecyanoacetates. Su, Weike; Yang, Bibo. Bulletin of the Chemical Society of Japan (2002), 75(10), 2221-2224.

17. Prolinamide functionalized polyacrylonitrile fiber with tunable linker length and surface microenvironment as efficient catalyst for Knoevenagel condensation and related multicomponent tandem reactions. Zhu, Hai; Xu, Gang; Du, Huimin; Zhang, Chenlu; Ma, Ning; Zhang, Wenqin. Journal of Catalysis (2019), 374, 217-229.

18. Synthesis and antifungal activities of novel polyheterocyclic spirooxindole derivatives. Wu, Jia-Shou; Zhang, Xue; Zhang, Ying-Lao; Xie, Jian-Wu. Organic \& Biomolecular Chemistry (2015), 13(17), 4967-4975. 
19. Synthesis of multifunctional polymer containing Ni-Pd NPs via thiol-ene reaction for one-pot cascade reactions. Javad Kalbasi, Roozbeh; Mesgarsaravi, Niloofar; Gharibi, Reza. Applied Organometallic Chemistry (2019), 33(4).

20. DBU-Promoted Cascade Annulation of Nitroarylcyclopropane-1,1-dicarbonitriles and 3-Aryl-2-cyanoacrylates: An Access to Highly Functionalized Cyclopenta[b]furan Derivatives. Qian, Siran; Xie, Zengyang; Liu, Jiaming; Li, Mingshuang; Wang, Shan; Luo, Naili; Wang, Cunde. Journal of Organic Chemistry (2018), 83(23), 14768-14776.

21. Metal-free synthesis of cyano acrylates via cyanuric chloride-mediated threecomponent reactions involving a cascade consists of Knoevenagel condensation/cyano hydration/esterification. Wan, Jie-Ping; Jing, Yanfeng; Liu, Yunyun; Sheng, Shouri. RSC Advances (2014), 4(109), 63997-64000.

22. A novel cyclodimerization of arylidenecyanoacetate promoted by samarium diiodide. Zhou, Longhu; Zhang, Yongmin. Tetrahedron Letters (1997), 38(46), 80638066.

23. Compartmentalization of incompatible polymers within metal-organic frameworks towards homogenization of heterogeneous hybrid catalysts for tandem reactions By Zhao, Jin-Hao; Yang, Yong; Che, Jin-Xin; Zuo, Jun; Li, Xiao-Hua; Hu, Yong-Zhou; Dong, Xiao-Wu; Gao, Liang; Liu, Xin-Yuan. Chemistry - A European Journal (2018), 24(39), 9903-9909.

24. Kharas GB, Christensen JL, Cichanski DJ, Goldman KE, Gordon CL, Knowles LM, et al. (2005) Novel copolymers of styrene and dialkoxy ring-substituted 
methyl 2-cyano-3-phenyl-2-propenoates. Journal of Macromolecular Science, Part A43(7): 989-994.

25. Kharas, GB, Shahbein A, Fabbri J, Fanter CE, Garcia DR, Ibarra MG, et al. (2014) Novel copolymers of styrene. 14. Some ring-disubstituted methyl 2-cyano-3phenyl-2-propenoates. J Macromol Sci A51(5): 394-398.

26. Kharas GB, Molina ES, Pierce EE, Cocjin SAB, Cruz C, Fair KM, et al. (2013) Novel copolymers of styrene. 4. Alkyl ring-substituted ethyl 2-cyano-3-phenyl-2propenoates. J Macromol Sci A50(2): 144-148.

27. Kharas GB, Delgado AA, Anderson NE, Bajor A, Colbert AC, Coleman A, et al. (2013) Novel copolymers of styrene. 6. Alkoxy ring-substituted ethyl 2-cyano-3phenyl-2-propenoates. J Macromol Sci A50(3): 276-280.

28. Kharas GB, Barros WMB, Affaneh A, Alyahya LA, Ansari AS, Asztalos MJ, et al. (2016) Novel copolymers of styrene. 5. Alkyl and alkoxy ring-disubstituted propyl 2-cyano-3-phenyl-2-propenoates. J Macromol Sci A53(11): 664-668.

29. Kharas GB, Feng H, Arendt LA, Belton SE, Edwards MQ, Franz EK, et al. (2015) Novel copolymers of styrene. 5. Methyl and methoxy ring-disubstituted butyl 2cyano-3-phenyl-2-propenoates. J Macromol Sci A52(12): 976-981.

30. Synthesis and Copolymerization of Halogen Phenyl-substituted Methyl 2-Cyano3-phenyl-2-propenoates with Styrene. K. Kim, C.A. Butler, M.R. Cisneros, S.L. Ryan, M.A. Schwartz, N.A. Lindquist, G.B. Kharas, J.V. DeFrancesco. Polymer Bull., 40, 361365 (1998).

31. Novel Copolymers of Styrene. 3. Halogen Ring-substituted Ethyl 2-Cyano-3-phenyl2-propenoates. G.B. Kharas, E.S. Molina, K.E. Abma, R.A. Ali, K.D. Bairaktaris, F.P. Biasiello, E.A. Cygan, J. Gibson, S. Haq, D.E. Hoag, K. Johnson, S.I. Jordan, M.B. Mathews, C.A. Shadduck, N. Tuzik. J.Macromol. Sci. A50 (2) 139-143 (2013). 
32. Novel Copolymers of Styrene. 4. Halogen Ring-Substituted Propyl 2-Cyano-3-

Phenyl-2-Propenoates. G.B. Kharas, V.A. Sloan-Lyon, J.D. Grannum, M.B. Gudger, M.L. Isai, L.A. Kopczynski, C.N. Lynch, H. M. Meyer, B.A. Roman, S.R. Schaefer, and L.F. Welk. J. Macromol. Sci. A53(11) 659-663 (2016).

33. Novel Copolymers of Styrene. 4. Halogen Ring-Substituted Butyl 2-Cyano-3Phenyl-2-Propenoates. G.B. Kharas, Y. Gao, E.E. Merriman, K.N. Parker, H.T. Patel, M.B. Raza, J. Sekhon, S.M. Stuckey, M. Styrczula, M.C. Ulloa, and W.F. Vital. J. Macromol. Sci. A52 (11) 887-891 (2015).

34. Synthesis and styrene copolymerization of novel halogen ring-substituted isobutyl 2-cyano-3-phenyl-2-propenoates. S.M. Rocus, M. Bailey, T.L. Brewer, D.O. Cline, J.K. Meighan, A.Z. Michalopoulos, Z.E. Moats, M.K. Mullen, T.J. Rendon, S.T. Rhodes, T. Samman, and G.B. Kharas. 260th ACS National Meeting \& Exposition, San Francisco, CA, USA, August 23-27 (2020), POLY-0079.

35 Synthesis and styrene copolymerization of novel trisubstituted ethylenes: 1. Alkyl ringsubstituted isopropyl 2-cyano-3-phenyl-2-propenoates. R.L. Pride, C.J. Anderson, M.S. Aelion, O.S. Barry, M. Berry, E. Blankemeyer, M.M. Bolton, A. Bravo, I. Chae, C.M. Franco, M. Galindo, W.S. Schjerven, G.B. Kharas. J. Macromol. Sci. 55 (4) 355-361(2018). 36. Smith MB, March J. Addition to carbon-hetero multiple bonds, In March's Advanced organic chemistry.: J. Wiley \& Sons, New York, 2001, Ch.16, 1225. https://doi.org/10.1002/9780470084960.ch16 